\title{
Application of OHAM-DJ to the System of Burgers' Equations
}

\author{
Fatheah Ahmad Alhendi', Bothayna Saleh Kashkari', Aisha Abdullah Alderremy ${ }^{2}$ \\ ${ }^{1}$ Department of Mathematics, King Abdulaziz University, Jeddah, Saudi Arabia \\ ${ }^{2}$ Department of Mathematics, King Khalid University, Abha, Saudi Arabia \\ Email: falhendi@kau.edu.sa,bkashkari@kau.edu.com, aaldramy@kku.edu.sa
}

Received 31 May 2016; accepted 8 August 2016; published 11 August 2016

Copyright (C) 2016 by authors and Scientific Research Publishing Inc.

This work is licensed under the Creative Commons Attribution International License (CC BY).

http://creativecommons.org/licenses/by/4.0/

(c) (7) Open Access

\begin{abstract}
In this paper, the system of Burgers' equations is solved by the optimal homotopy asymptotic method with Daftardar-Jafari polynomials OHAM-DJ. Two numerical examples are illustrated the efficient of this methods for solving the system of Burgers' equations.
\end{abstract}

\section{Keywords}

\section{Burgers' Equations, The Optimal Homotopy Asymptotic Method, Daftardar-Jafari Polynomials}

\section{Introduction}

The Burgers equation was first presented by Bateman [1] and treated later by J. M. Burgers (1895-1981) then it is widely named as Burgers' equation [2]. Burgers' equation is nonlinear partial differential equation of second order which is used in various fields of physical phenomena such as boundary layer behaviour, shock weave formation, turbulence, the weather problem, mass transport, traffic flow and acoustic transmission [3] [4]. In addition, the two dimentional Burgers' equations have played an important role in many physical applications such as investigating the shallow water waves and modeling of gas dynamics [5] [6]. In order to a great applications for burgers' equations many researchers have been interested in solving it by various techniques. Analytic solution of one dimensional Burgers' equation are get by many standard methods such as Backland transformation method, differential transformation method and tanh-coth method [6], while an analytical solution of two dimensional Burgers' equations was first presented by Fletcher using the Hopf-Cole transformation [7]. The finite difference, finite element, spectral methods, Adomian decomposition method, the variational iteration method, homotopy perturbation method HPM and Eulerian-Lagrangian method gave an numerical solution of Burgers' equations [3] [8]-[15].

Recently, the OHAM was proposed by Marinca and Herisaun [16]-[19]. OHAM is independent of the 
existence of a embedding parameter in the problem then overcome the limitations of perturbation technique. However, OHAM is the most generalized form of HPM as it uses a general auxiliary function H(p). This method has been studied by a number of researchers for solving linear and nonlinear partial differential equations [20]-[23]. In [24]-[27] proved OHAM is more efficient to solve Burgers' equations. In 2006, a new method by Daftardar-Gejji and Jafari for solving nonlinear functional appeared [28]. Convergence of it has been proved in [29]. This method is named later as Daftardar-Jafari method DJM in [30]. J. Ali et al. used DJM in the OHAM for solving non-linear differential equations and they named this method as OHAM with DJ polynomials OHAM-DJ [30] [31]. In 2016, OHAM-DJ has been used to solve linear and nonlinear Klein-Gordon equations [32]. The motive of this paper is to show the efficiency of OHAM-DJ for solving the system of Burger's equations. We consider the system of Burger's equations as the following [11]:

$$
\begin{aligned}
& \frac{\partial u}{\partial t}+u \frac{\partial u}{\partial x}+v \frac{\partial u}{\partial y}=\frac{1}{R}\left(\frac{\partial^{2} u}{\partial x^{2}}+\frac{\partial^{2} u}{\partial y^{2}}\right) \\
& \frac{\partial v}{\partial t}+u \frac{\partial v}{\partial x}+v \frac{\partial v}{\partial y}=\frac{1}{R}\left(\frac{\partial^{2} v}{\partial x^{2}}+\frac{\partial^{2} v}{\partial y^{2}}\right)
\end{aligned}
$$

with the initial conditions:

$$
\begin{aligned}
& u(x, y, 0)=f(x, y), \quad(x, y) \in \Omega \\
& v(x, y, 0)=g(x, y),(x, y) \in \Omega
\end{aligned}
$$

and the boundary conditions:

$$
\begin{aligned}
& u(x, y, t)=f_{1}(x, y, t), \quad x, y \in \Gamma, t>0 \\
& v(x, y, t)=f_{2}(x, y, t), \quad x, y \in \Gamma, t>0
\end{aligned}
$$

where $\Omega=\{(x, y) \mid a \leq x \leq b, a \leq y \leq b\}$ and $\Gamma$ is its boundary, $u(x, y, t)$ and $v(x, y, t)$ are the velocity components to be determined, $f, g, f_{1}$ and $f_{2}$ are known functions and $R$ is the Reynolds number.

This paper is organized into three sections. In Section 2 methodology of OHAM-DJ is presented. In Section 3 application of this method is solved and absolute error of approximate solutions of proposed method is compared with exact solutions. In all cases the proposed method yields better results.

\section{Methodology of OHAM-DJ}

Consider (1.1) and let

$$
\begin{aligned}
& \beta_{1}\left(u(x, y, t), \frac{\partial u(x, y, t)}{\partial t}\right)=0 \\
& \beta_{2}\left(v(x, y, t), \frac{\partial v(x, y, t)}{\partial t}\right)=0
\end{aligned}
$$

where $\beta_{1}, \beta_{2}$ are boundary operators.

According to the basic idea of OHAM [16], we can construct the optimal homotopy:

$$
\begin{aligned}
& U(x, y, t ; p): \Omega \times[0,1] \rightarrow R \\
& V(x, y, t ; p): \Omega \times[0,1] \rightarrow R
\end{aligned}
$$

which satisfies

$$
\begin{aligned}
& (1-p)\left(\frac{\partial U}{\partial t}\right)-H_{1}(P)\left(\frac{\partial U}{\partial t}+U \frac{\partial U}{\partial x}+V \frac{\partial U}{\partial y}-\frac{1}{R}\left(\frac{\partial^{2} U}{\partial x^{2}}+\frac{\partial^{2} U}{\partial y^{2}}\right)\right)=0 \\
& (1-p)\left(\frac{\partial V}{\partial t}\right)-H_{2}(P)\left(\frac{\partial V}{\partial t}+U \frac{\partial V}{\partial x}+V \frac{\partial V}{\partial y}-\frac{1}{R}\left(\frac{\partial^{2} V}{\partial x^{2}}+\frac{\partial^{2} V}{\partial y^{2}}\right)\right)=0
\end{aligned}
$$




$$
\begin{aligned}
& \beta_{1}\left(U(x, y, t ; p), \frac{\partial U(x, y, t ; p)}{\partial t}\right)=0 \\
& \beta_{2}\left(V(x, y, t ; p), \frac{\partial V(x, y, t ; p)}{\partial t}\right)=0
\end{aligned}
$$

where $p \in[0,1]$ is an embedding parameter while $u_{0}$ and $v_{0}$ is initial approximation of Equation (1.1) which satisfies the boundary condition, $H_{1}(p)$ and $H_{2}(p)$ are nonzero auxiliary functions for $p \neq 0$, $H_{1}(0)=0$ and $H_{2}(0)=0$. Clearly, When $p=0$ and $p=1$, it holds that $U(x, y, t ; 0)=u_{0}(x, y, t)$, $V(x, y, t ; 0)=v_{0}(x, y, t)$ and $U(x, y, t ; 1)=u(x, y, t), V(x, y, t ; 1)=v(x, y, t)$ respectively. Therefore, as $p$ change from 0 to 1 , the solution $U(x, y, t ; p)$ and $V(x, y, t ; p)$ varies from $u_{0}(x, y)$ to $u(x, y, t)$ and $v_{0}(x, y)$ to $v(x, y, t)$ respectively, where the initial approximations $u_{0}(x, y)$ and $v_{0}(x, y)$ are obtained from (2.3) and (2.4). Now, choosing The auxiliary functions $H_{1}(p)$ and $H_{2}(p)$ as the form

$$
\begin{aligned}
& H_{1}(p)=p c_{1}+p^{2} c_{2}+p^{3} c_{3}+\cdots \\
& H_{2}(p)=p d_{1}+p^{2} d_{2}+p^{3} d_{3}+\cdots
\end{aligned}
$$

where, $c_{i}, d_{i}, i=1,2,3, \cdots$ are constants to be determined later. Assume that the solutions of (1.1) has the form:

$$
\begin{aligned}
& U=u_{0}(x, y)+\sum_{i=1}^{\infty} p^{i} u_{i}\left(x, y, t ; c_{i}\right) \\
& V=v_{0}(x, y)+\sum_{i=1}^{\infty} p^{i} v_{i}\left(x, y, t ; d_{i}\right)
\end{aligned}
$$

The nonlinear terms

$$
\begin{aligned}
& A=U \frac{\partial U}{\partial x}, B=V \frac{\partial U}{\partial y} \\
& C=U \frac{\partial V}{\partial y}, \quad D=V \frac{\partial V}{\partial y}
\end{aligned}
$$

are decomposed as

$$
\begin{aligned}
& A=A\left(u_{0}\right)+p\left[A\left(u_{0}+u_{1}\right)-A\left(u_{0}\right)\right]+p^{2}\left[A\left(u_{0}+u_{1}+u_{2}\right)-A\left(u_{0}+u_{1}\right)\right]+\cdots \\
& B=B\left(u_{0}\right)+p\left[B\left(u_{0}+u_{1}\right)-B\left(u_{0}\right)\right]+p^{2}\left[B\left(u_{0}+u_{1}+u_{2}\right)-B\left(u_{0}+u_{1}\right)\right]+\cdots \\
& C=C\left(u_{0}\right)+p\left[C\left(u_{0}+u_{1}\right)-C\left(u_{0}\right)\right]+p^{2}\left[C\left(u_{0}+u_{1}+u_{2}\right)-C\left(u_{0}+u_{1}\right)\right]+\cdots \\
& D=D\left(u_{0}\right)+p\left[D\left(u_{0}+u_{1}\right)-D\left(u_{0}\right)\right]+p^{2}\left[D\left(u_{0}+u_{1}+u_{2}\right)-D\left(u_{0}+u_{1}\right)\right]+\cdots
\end{aligned}
$$

where $F_{l}\left(u_{0}\right),\left[F_{l}\left(u_{0}+u_{1}\right)-F_{l}\left(u_{0}\right)\right],\left[F_{l}\left(u_{0}+u_{1}+u_{2}\right)-F_{l}\left(u_{0}+u_{1}\right)\right], \cdots, l=1, \cdots, 4$ are (DJ) polynomials, $A=F_{1}, B=F_{2}, C=F_{3}, D=F_{4}$. For simplicity these polynomials are expressed as:

$$
\begin{aligned}
A_{0} & =A\left(u_{0}\right) \\
A_{1} & =A\left(u_{0}+u_{1}\right)-A\left(u_{0}\right) \\
A_{2} & =A\left(u_{0}+u_{1}+u_{2}\right)-A\left(u_{0}+u_{1}\right) \\
& \vdots \\
A_{m} & =A\left(\sum_{j=0}^{m} u_{j}\right)-A\left(\sum_{j=0}^{m-1} u_{j}\right) \\
A & =A_{0}+\sum_{k=1}^{\infty} p^{k} A_{k}
\end{aligned}
$$

Then, 


$$
A=A_{0}+\sum_{k=1}^{\infty} p^{k} A_{k}, B=B_{0}+\sum_{k=1}^{\infty} p^{k} B_{k}, C=C_{0}+\sum_{k=1}^{\infty} p^{k} C_{k}, D=D_{0}+\sum_{k=1}^{\infty} p^{k} D_{k}
$$

Substiting, (2.5),(2.6), (2.7) and (2.9) into (2.3), and comparing the coefficients of like powers of $p$, we get

$$
\begin{aligned}
& p^{0}:\left\{\begin{array}{l}
\frac{\partial u_{0}}{\partial t}=0, \beta_{1}\left(u_{0}, \frac{\partial u_{0}}{\partial t}\right)=0 \\
\frac{\partial v_{0}}{\partial t}=0, \beta_{2}\left(v_{0}, \frac{\partial v_{0}}{\partial t}\right)=0
\end{array}\right. \\
& p^{1}:\left\{\begin{array}{l}
\frac{\partial u_{1}}{\partial t}=\frac{\partial u_{0}}{\partial t}+c_{1}\left(\frac{\partial u_{0}}{\partial t}+u_{0} \frac{\partial u_{0}}{\partial x}+v_{0} \frac{\partial u_{0}}{\partial y}-\frac{\partial^{2} u_{0}}{\partial x^{2}}-\frac{\partial^{2} u_{0}}{\partial y^{2}}\right), \beta_{1}\left(u_{1}, \frac{\partial u_{1}}{\partial t}\right)=0 \\
\frac{\partial v_{1}}{\partial t}=\frac{\partial v_{0}}{\partial t}+d_{1}\left(\frac{\partial v_{0}}{\partial t}+u_{0} \frac{\partial v_{0}}{\partial x}+v_{0} \frac{\partial v_{0}}{\partial y}-\frac{\partial^{2} v_{0}}{\partial x^{2}}-\frac{\partial^{2} v_{0}}{\partial y^{2}}\right), \beta_{2}\left(v_{1}, \frac{\partial v_{1}}{\partial t}\right)=0
\end{array}\right. \\
& \int \frac{\partial u_{2}}{\partial t}=\frac{\partial u_{1}}{\partial t}+c_{1}\left(\frac{\partial u_{1}}{\partial t}+u_{0} \frac{\partial u_{1}}{\partial x}+u_{1} \frac{\partial u_{0}}{\partial x}+u_{1} \frac{\partial u_{1}}{\partial x}+v_{0} \frac{\partial u_{1}}{\partial y}+v_{1} \frac{\partial u_{0}}{\partial y}+v_{1} \frac{\partial u_{1}}{\partial y}-\frac{\partial^{2} u_{1}}{\partial x^{2}}-\frac{\partial^{2} u_{1}}{\partial y^{2}}\right) \\
& +c_{2}\left(\frac{\partial u_{0}}{\partial t}+u_{0} \frac{\partial u_{0}}{\partial x}+v_{0} \frac{\partial u_{0}}{\partial y}-\frac{\partial^{2} u_{0}}{\partial x^{2}}-\frac{\partial^{2} u_{0}}{\partial y^{2}}\right), \beta_{1}\left(u_{2}, \frac{\partial u_{2}}{\partial t}\right)=0 \\
& p^{2}:\left\{\begin{array}{l}
\frac{\partial v_{2}}{\partial t}=\frac{\partial v_{1}}{\partial t}+d_{1}\left(\frac{\partial v_{1}}{\partial t}+u_{0} \frac{\partial v_{1}}{\partial x}+u_{1} \frac{\partial v_{0}}{\partial x}+u_{1} \frac{\partial v_{1}}{\partial x}+v_{0} \frac{\partial v_{1}}{\partial y}+v_{1} \frac{\partial v_{0}}{\partial y}+v_{1} \frac{\partial v_{1}}{\partial y}-\frac{\partial^{2} v_{1}}{\partial x^{2}}-\frac{\partial^{2} v_{1}}{\partial y^{2}}\right)
\end{array}\right. \\
& +d_{2}\left(\frac{\partial v_{0}}{\partial t}+u_{0} \frac{\partial v_{0}}{\partial x}+v_{0} \frac{\partial v_{0}}{\partial y}-\frac{\partial^{2} v_{0}}{\partial x^{2}}-\frac{\partial^{2} v_{0}}{\partial y^{2}}\right), \beta_{2}\left(v_{2}, \frac{\partial v_{2}}{\partial t}\right)=0
\end{aligned}
$$

The convergence of (2.6) depend upon the auxiliary constants $c_{i}$ and $d_{i}$, which known convergence control parameters or optimal convergence control parameters [16], if it is convergent at $p=1$ we have

$$
\begin{aligned}
& \tilde{u}\left(x, y, t ; c_{i}\right)=u_{0}(x, y)+u_{1}\left(x, y, t ; c_{1}\right)+u_{2}\left(x, y, t ; c_{1}, c_{2}\right)+\cdots \\
& \tilde{v}\left(x, y, t ; d_{i}\right)=v_{0}(x, y)+v_{1}\left(x, y, t ; d_{1}\right)+v_{2}\left(x, y, t ; d_{1}, d_{2}\right)+\cdots
\end{aligned}
$$

Substituting (2.11) into (1.1) we get the residuals $\mathcal{R}_{1}\left(x, y, t ; c_{i}, d_{i}\right)$ and $\mathcal{R}_{2}\left(x, y, t ; c_{i}, d_{i}\right), i=1,2, \cdots, n$ these parameters can be optimal identified by various methods [16] [20] [33]. Optimization method is one of theses methods to find out the optimal convergence control parameters by means of the minimum of the squared residuals.

\section{Numerical Examples}

In this section, two numerical examples are used to prove the efficiency and the accuracy of the method which we proposed for the system of Burgers' equations.

\subsection{Example 1}

Consider the system of two dimensional of Burgers' equations with the initial conditions as following [34]

$$
\begin{aligned}
& \frac{\partial u}{\partial t}+u \frac{\partial u}{\partial x}+v \frac{\partial u}{\partial y}=\varepsilon\left(\frac{\partial^{2} u}{\partial x^{2}}+\frac{\partial^{2} u}{\partial y^{2}}\right) \\
& \frac{\partial v}{\partial t}+u \frac{\partial v}{\partial x}+v \frac{\partial v}{\partial y}=\varepsilon\left(\frac{\partial^{2} v}{\partial x^{2}}+\frac{\partial^{2} v}{\partial y^{2}}\right)
\end{aligned}
$$


with the initial conditions:

$$
\begin{aligned}
& u(x, y, 0)=\frac{3}{4}-\frac{1}{4\left(1+\mathrm{e}^{\frac{y-x}{8 \varepsilon}}\right)} \\
& v(x, y, 0)=\frac{3}{4}+\frac{1}{4\left(1+\mathrm{e}^{\frac{y-x}{8 \varepsilon}}\right)}
\end{aligned}
$$

The exact solutions are

$$
\begin{aligned}
& u^{*}(x, y, t)=\frac{3}{4}-\frac{1}{4\left(1+\mathrm{e}^{\frac{4 y-4 x-t}{32 \varepsilon}}\right)} \\
& v^{*}(x, y, t)=\frac{3}{4}+\frac{1}{4\left(1+\mathrm{e}^{\frac{4 y-4 x-t}{32 \varepsilon}}\right)}
\end{aligned}
$$

Accordance to the methodology of OHAM-DJ, $\varepsilon=1$

$p^{0}:\left\{\begin{array}{l}\frac{\partial u_{0}}{\partial t}=0, \beta_{1}\left(u_{0}, \frac{\partial u_{0}}{\partial t}\right)=0 \\ \frac{\partial v_{0}}{\partial t}=0, \beta_{2}\left(v_{0}, \frac{\partial v_{0}}{\partial t}\right)=0\end{array}\right.$

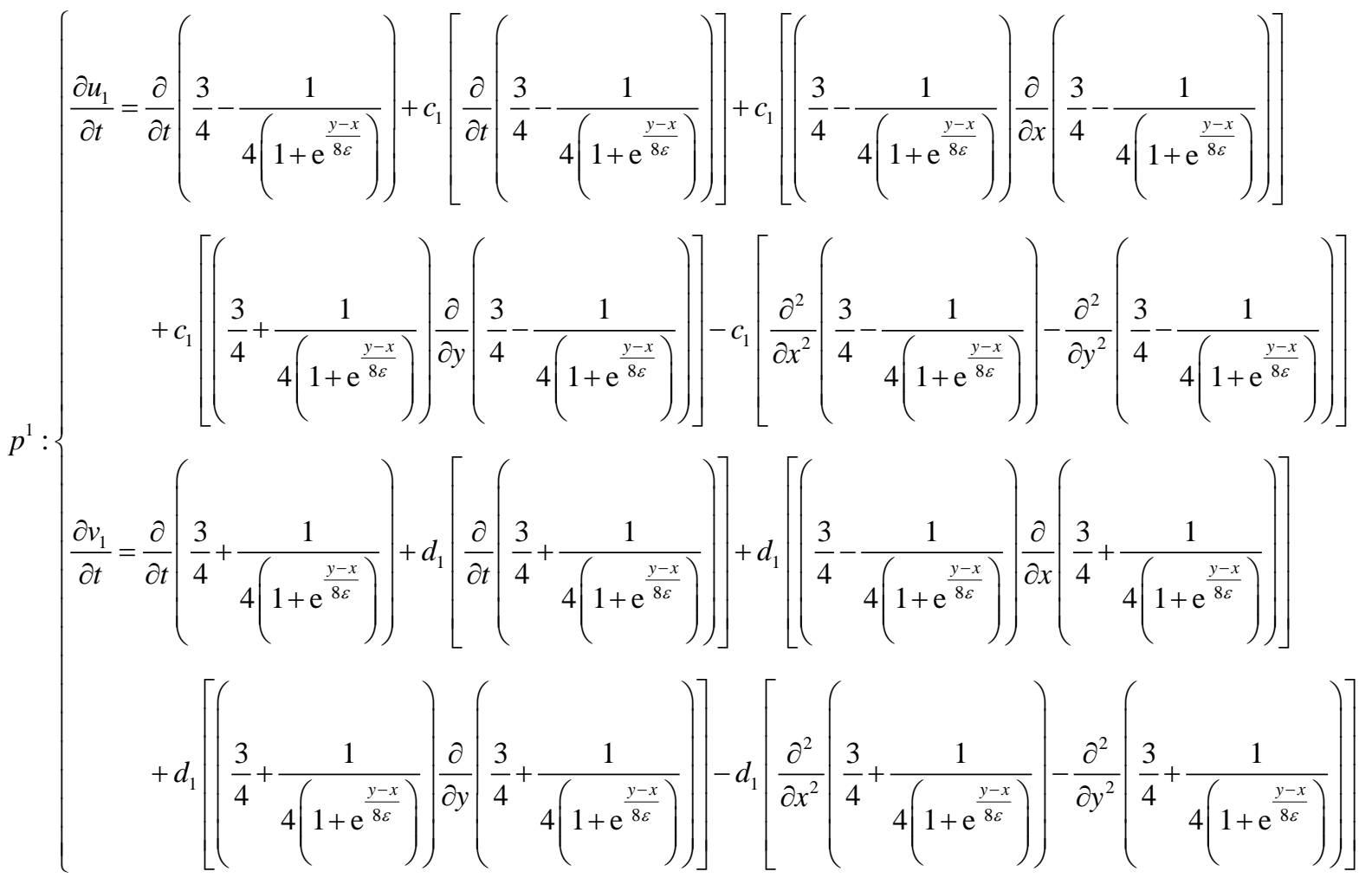


Their solutions are

$$
\begin{aligned}
& \left\{\begin{array}{l}
u_{0}(x, y)=\frac{3}{4}-\frac{1}{4\left(1+\mathrm{e}^{\frac{y-x}{8 \varepsilon}}\right)} \\
v_{0}(x, y)=\frac{3}{4}+\frac{1}{4\left(1+\mathrm{e}^{\frac{y-x}{8 \varepsilon}}\right)} \\
u_{1}\left(x, y, t ; c_{1}\right)=\frac{1}{128} \frac{c_{1} \mathrm{e}^{\frac{1}{8} y-\frac{1}{8} x} t}{\left(1+\mathrm{e}^{\frac{1}{8} y-\frac{1}{8} x}\right)^{2}} \\
v_{1}\left(x, y, t ; d_{1}\right)=-\frac{1}{128} \frac{d_{1} \mathrm{e}^{\frac{1}{8} y-\frac{1}{8} x} t}{\left(1+\mathrm{e}^{\frac{1}{8} y-\frac{1}{8} x}\right)^{2}}
\end{array}\right.
\end{aligned}
$$

Then,

$$
\begin{aligned}
& \tilde{u}\left(x, y, t ; c_{i}\right)=\frac{3}{4}-\frac{1}{4\left(1+\mathrm{e}^{\frac{1}{8} y-\frac{1}{8} x}\right)}+\frac{1}{128} \frac{c_{1} \mathrm{e}^{\frac{1}{8} y-\frac{1}{8} x} t}{\left(1+\mathrm{e}^{\frac{1}{8} y-\frac{1}{8} x}\right)^{2}}+\cdots \\
& \tilde{v}\left(x, y, t ; d_{i}\right)=\frac{3}{4}+\frac{1}{4\left(1+\mathrm{e}^{\frac{1}{8} y-\frac{1}{8} x}\right)}-\frac{1}{128} \frac{d_{1} \mathrm{e}^{\frac{1}{8} y-\frac{1}{8} x} t}{\left(1+\mathrm{e}^{\frac{1}{8} y-\frac{1}{8} x}\right)^{2}}+\cdots
\end{aligned}
$$

By substituting (3.6) into (3.1) we get the residuals and using the optimization method we have computed that $c_{1}=-0.999927000502526$ and $d_{1}=1.00007801756198$. Finally, putting the values of $c_{1}$ and $d_{1}$ into (3.6), to get the approximate solutions (Tables 1-3, Figure 1 and Figure 2).

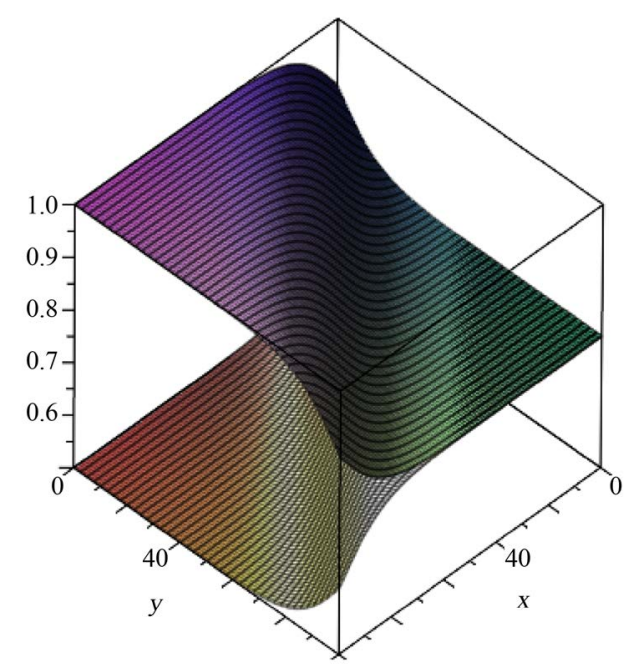

Figure 1. Approximation solutions by OHAM-DJ of example $1, t=0.01, \quad \varepsilon=1$. 


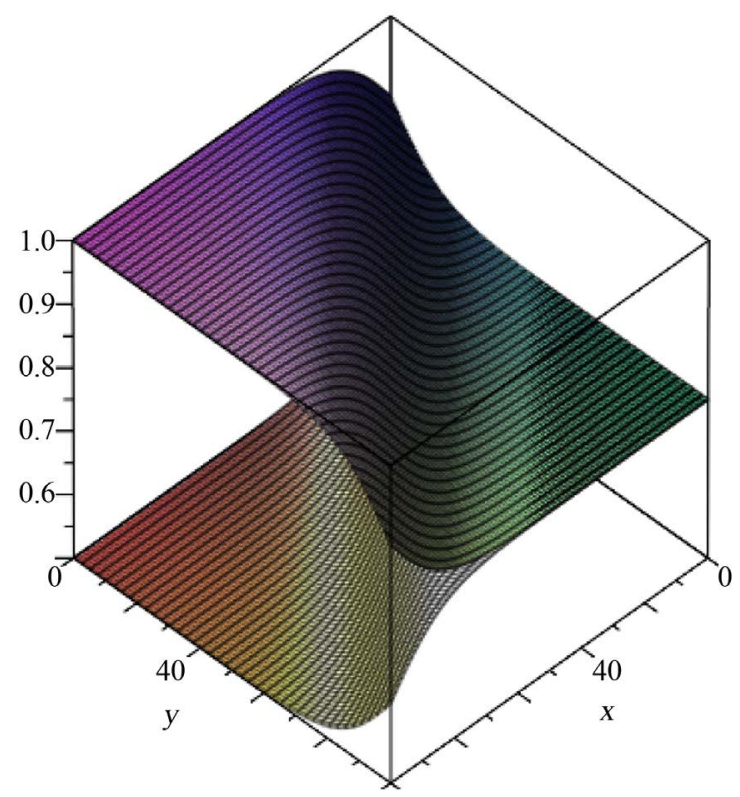

Figure 2. Exact solutions of example $1, t=0.01, \varepsilon=1$.

Table 1. Comparison of OHAM-DJ solutions with exact solutions at mesh point $x=2, y=1$ (example 1).

\begin{tabular}{ccccccc}
\hline $\mathrm{t}$ & $u^{*}(x, y, t)$ & $u(x, y, t)$ & $\left|u^{*}-u\right|$ & $v^{*}(x, y, t)$ & $v(x, y, t)$ & $\left|v^{*}-v\right|$ \\
\hline 0.01 & 0.6171782017 & 0.6171782030 & $1.3 \times 10^{-9}$ & 0.8828217983 & 0.8828217970 & $1.3 \times 10^{-9}$ \\
0.02 & 0.6171587471 & 0.6171587492 & $2.1 \times 10^{-9}$ & 0.8828412529 & 0.8828412508 & $2.1 \times 10^{-9}$ \\
0.03 & 0.6171392929 & 0.6171392955 & $2.6 \times 10^{-9}$ & 0.8828607071 & 0.8828607045 & $2.6 \times 10^{-9}$ \\
0.04 & 0.6171198391 & 0.6171198418 & $2.7 \times 10^{-9}$ & 0.8828801609 & 0.8828801582 & $2.7 \times 10^{-9}$ \\
0.05 & 0.6171003857 & 0.6171003880 & $2.3 \times 10^{-9}$ & 0.8828996143 & 0.8828996120 & $2.3 \times 10^{-9}$ \\
0.06 & 0.6170809326 & 0.6170809343 & $1.7 \times 10^{-9}$ & 0.8829190674 & 0.8829190657 & $1.7 \times 10^{-9}$ \\
0.07 & 0.6170614799 & 0.6170614806 & $7 \times 10^{-10}$ & 0.8829385201 & 0.8829385194 & $7 \times 10^{-10}$ \\
0.08 & 0.6170420276 & 0.6170420268 & $8 \times 10^{-10}$ & 0.8829579724 & 0.8829579732 & $8 \times 10^{-10}$ \\
0.09 & 0.6170225757 & 0.6170225731 & $2.6 \times 10^{-9}$ & 0.8829774243 & 0.8829774269 & $2.6 \times 10^{-9}$ \\
\hline
\end{tabular}

\subsection{Example 2}

We consider the following two-dimensional Burgers' equations [34]

$$
u_{t}+u\left(u_{x}+u_{y}\right)=\varepsilon\left(u_{x x}+u_{y y}\right)
$$

On square domain $D:[0,2] \times[0,2]$, with the initial condition:

$$
u(x, y, 0)=\frac{1}{1+\mathrm{e}^{x+y / 2 \varepsilon}},(x, y) \in D
$$

for which the exact solution is $u^{*}(x, y, t)=\frac{1}{1+\mathrm{e}^{x+y-t / 2 \varepsilon}},(x, y) \in D, t \geq 0$. Where the $u(x, y, t)$ and $v(x, y, t)$ in (1.1) are symmetry in this example, $u(x, y, t)=v(x, y, t)$ and the initial condition are symmetry also. 
Table 2. Comparison of OHAM-DJ solutions with exact solutions at mesh point $x=1, y=2$ (example 1 ).

\begin{tabular}{ccccccc}
\hline $\mathrm{t}$ & $u^{*}(x, y, t)$ & $u(x, y, t)$ & $\left|u^{*}-u\right|$ & $v^{*}(x, y, t)$ & $v(x, y, t)$ & $\left|v^{*}-v\right|$ \\
\hline 0.01 & 0.6327828880 & 0.6327828896 & $1.6 \times 10^{-9}$ & 0.8672171120 & 0.8672171104 & $1.6 \times 10^{-9}$ \\
0.02 & 0.6327634323 & 0.6327634358 & $3.5 \times 10^{-9}$ & 0.8672365677 & 0.8672365642 & $3.5 \times 10^{-9}$ \\
0.03 & 0.6327439762 & 0.6327439821 & $5.9 \times 10^{-9}$ & 0.8672560238 & 0.8672560179 & $5.9 \times 10^{-9}$ \\
0.04 & 0.6327245197 & 0.6327245284 & $8.7 \times 10^{-9}$ & 0.8672754803 & 0.8672754716 & $8.7 \times 10^{-9}$ \\
0.05 & 0.6327050628 & 0.6327050746 & $1.18 \times 10^{-8}$ & 0.8672949372 & 0.8672949254 & $1.18 \times 10^{-8}$ \\
0.06 & 0.6326856056 & 0.6326856209 & $1.53 \times 10^{-8}$ & 0.8673143944 & 0.8673143791 & $1.53 \times 10^{-8}$ \\
0.07 & 0.6326661480 & 0.6326661672 & $1.92 \times 10^{-8}$ & 0.8673338520 & 0.8673338328 & $1.92 \times 10^{-8}$ \\
0.08 & 0.6326466900 & 0.6326467134 & $2.34 \times 10^{-8}$ & 0.8673533100 & 0.8673532866 & $2.34 \times 10^{-8}$ \\
0.09 & 0.6326272317 & 0.6326272597 & $2.80 \times 10^{-8}$ & 0.8673727683 & 0.8673727403 & $2.80 \times 10^{-8}$ \\
0.10 & 0.6326077730 & 0.6326078060 & $3.30 \times 10^{-8}$ & 0.8673922270 & 0.8673921940 & $3.30 \times 10^{-8}$ \\
\hline
\end{tabular}

Table 3. Comparison of OHAM-DJ solutions with exact solutions at mesh point $x=1.5, y=2$ (example 1 ).

\begin{tabular}{ccccccc}
\hline $\mathrm{t}$ & $u^{*}(x, y, t)$ & $u(x, y, t)$ & $\left|u^{*}-u\right|$ & $v^{*}(x, y, t)$ & $v(x, y, t)$ & 0.8711145319 \\
0.01 & 0.6288854666 & 0.6288854681 & $1.5 \times 10^{-9}$ & 0.8711145334 & $0.5 \times 10^{-9}$ \\
0.02 & 0.6288659542 & 0.6288659574 & $3.2 \times 10^{-9}$ & 0.8711340458 & 0.8711340426 \\
0.03 & 0.6288464415 & 0.6288464466 & $5.1 \times 10^{-9}$ & 0.8711535585 & 0.8711535534 \\
0.04 & 0.6288269287 & 0.6288269358 & $7.1 \times 10^{-9}$ & 0.8711730713 & 0.8711730642 \\
0.05 & 0.6288074156 & 0.6288074251 & $9.5 \times 10^{-9}$ & 0.8711925844 & 0.8711925749 \\
0.06 & 0.6287879024 & 0.6287879143 & $1.19 \times 10^{-8}$ & 0.8712120976 & 0.8712120857 \\
0.07 & 0.6287683890 & 0.6287684035 & $1.45 \times 10^{-8}$ & 0.8712316110 & 0.8712315965 \\
0.08 & 0.6287488754 & 0.6287488928 & $1.74 \times 10^{-8}$ & 0.8712511246 & 0.8712511072 \\
0.09 & 0.6287293616 & 0.6287293820 & $2.04 \times 10^{-8}$ & 0.8712706384 & 0.8712706180 \\
0.10 & 0.6287098477 & 0.6287098713 & $2.36 \times 10^{-8}$ & 0.8712901523 & 0.8712901287 \\
\hline
\end{tabular}

$$
\begin{aligned}
& p^{0}:\left\{\begin{array}{l}
\frac{\partial u_{0}}{\partial t}=0, \beta_{1}\left(u_{0}, \frac{\partial u_{0}}{\partial t}\right)=0 \\
\frac{\partial v_{0}}{\partial t}=0, \beta_{2}\left(v_{0}, \frac{\partial v_{0}}{\partial t}\right)=0
\end{array}\right. \\
& \int \frac{\partial u_{1}}{\partial t}=\frac{\partial}{\partial t}\left(\frac{1}{1+\mathrm{e}^{x+y / 2 \varepsilon}}\right)+c_{1}\left[\frac{\partial}{\partial t}\left(\frac{1}{1+\mathrm{e}^{x+y / 2 \varepsilon}}\right)\right] \\
& +c_{1}\left[\frac{1}{1+\mathrm{e}^{x+y / 2 \varepsilon}} \frac{\partial}{\partial x}\left(\frac{1}{1+\mathrm{e}^{x+y / 2 \varepsilon}}\right)+\frac{1}{1+\mathrm{e}^{x+y / 2 \delta}} \frac{\partial}{\partial y}\left(\frac{1}{1+\mathrm{e}^{x+y / 2 \varepsilon}}\right)\right] \\
& -c_{1}\left[\varepsilon \frac{\partial^{2}}{\partial x^{2}}\left(\frac{1}{1+\mathrm{e}^{x+y / 2 \varepsilon}}\right)-\varepsilon \frac{\partial^{2}}{\partial y^{2}}\left(\frac{1}{1+\mathrm{e}^{x+y / 2 \varepsilon}}\right)\right] \\
& p^{1}:\left\{\frac{\partial v_{1}}{\partial t}=\frac{\partial}{\partial t}\left(\frac{1}{1+\mathrm{e}^{x+y / 2 \varepsilon}}\right)+c_{1}\left[\frac{\partial}{\partial t}\left(\frac{1}{1+\mathrm{e}^{x+y / 2 \varepsilon}}\right)\right]\right. \\
& +c_{1}\left[\frac{1}{1+\mathrm{e}^{x+y / 2 \varepsilon}} \frac{\partial}{\partial x}\left(\frac{1}{1+\mathrm{e}^{x+y / 2 \varepsilon}}\right)+\frac{1}{1+\mathrm{e}^{x+y / 2 \varepsilon}} \frac{\partial}{\partial y}\left(\frac{1}{1+\mathrm{e}^{x+y / 2 \varepsilon}}\right)\right] \\
& -c_{1}\left[\varepsilon \frac{\partial^{2}}{\partial x^{2}}\left(\frac{1}{1+\mathrm{e}^{x+y / 2 \varepsilon}}\right)-\varepsilon \frac{\partial^{2}}{\partial y^{2}}\left(\frac{1}{1+\mathrm{e}^{x+y / 2 \varepsilon}}\right)\right]
\end{aligned}
$$


Their solutions are

$$
\begin{aligned}
& \left\{\begin{array}{l}
u_{0}(x, y)=\frac{1}{1+\mathrm{e}^{x+y / 2 \varepsilon}} \\
v_{0}(x, y)=\frac{1}{1+\mathrm{e}^{x+y / 2 \varepsilon}} \\
\left\{\begin{array}{l}
u_{1}\left(x, y, t ; c_{1}\right)=c_{1}\left[-\frac{1}{2} \frac{\mathrm{e}^{(x+y) / 2 \varepsilon}}{\varepsilon\left(1+\mathrm{e}^{(x+y) / 2 \varepsilon}\right)^{2}}\right] t \\
v_{1}\left(x, y, t ; c_{1}\right)=c_{1}\left[-\frac{1}{2} \frac{\mathrm{e}^{(x+y) / 2 \varepsilon}}{\varepsilon\left(1+\mathrm{e}^{(x+y) / 2 \varepsilon}\right)^{2}}\right] t
\end{array}\right. \\
\tilde{u}\left(x, y, t ; c_{i}\right)=\frac{1}{1+\mathrm{e}^{x+y / 2 \varepsilon}}+c_{1}\left[-\frac{1}{2} \frac{\mathrm{e}^{(x+y) / 2 \varepsilon}}{\varepsilon\left(1+\mathrm{e}^{(x+y) / 2 \varepsilon}\right)^{2}}\right] t+\cdots
\end{array}\right] \\
& \tilde{v}\left(x, y, t ; c_{i}\right)=\frac{1}{1+\mathrm{e}^{x+y / 2 \varepsilon}}+c_{1}\left[-\frac{1}{2} \frac{\mathrm{e}^{(x+y) / 2 \varepsilon}}{\varepsilon\left(1+\mathrm{e}^{(x+y) / 2 \varepsilon}\right)^{2}}\right] t+\cdots
\end{aligned}
$$

Substituting (3.11) into (3.7) we get the residuals and using the optimization method we have computed that $c_{1}=-1.01431980619957$. Finally, putting the values of $c_{1}$ into (3.11) to get the approximate solutions (Table 4 and Table 5, Figure 3 and Figure 4).

\section{Conclusion}

In this work, the OHAM-DJ is applied to obtain numerical solutions of the system of Burgers' equations. The method is efficient and easy to implement where the first or second order solutions rapidly converges to the exact solutions. Furthermore, OHAM-DJ does not need any discretization in time or in space. Thus the solutions of system of Burgers' equations are not influenced by computer round off errors. The method can be easily

Table 4. Comparison of OHAM-DJ solutions with exact solutions at mesh point $x=1, y=1, \quad \varepsilon=0.1$ (example 2).

\begin{tabular}{cccc}
\hline $\mathrm{t}$ & $u^{*}(x, y, t)=v^{*}(x, y, t)$ & $u(x, y, t)=v(x, y, t)$ & $\left|u^{*}-u\right|=\left|v^{*}-v\right|$ \\
\hline 0.01 & 0.00004772535612 & 0.00004770016206 & $2.519406 \times 10^{-8}$ \\
0.02 & 0.00005017216468 & 0.00005000245540 & $1.6970928 \times 10^{-7}$ \\
0.03 & 0.00005274441090 & 0.00005230474874 & $4.3966216 \times 10^{-7}$ \\
0.04 & 0.00005544852472 & 0.00005460704209 & $8.4148263 \times 10^{-7}$ \\
0.05 & 0.00005829126566 & 0.00005690933545 & $1.38193021 \times 10^{-6}$ \\
0.06 & 0.00006127973961 & 0.00005921162878 & $2.06811083 \times 10^{-6}$ \\
0.07 & 0.00006442141667 & 0.00006151392213 & $2.90749454 \times 10^{-6}$ \\
0.08 & 0.00006772414960 & 0.00006381621547 & $3.90793413 \times 10^{-6}$ \\
0.09 & 0.00007119619382 & 0.00006611850883 & $5.07768499 \times 10^{-6}$ \\
0.10 & 0.00007484622751 & 0.00006842080217 & $6.42542534 \times 10^{-6}$ \\
\hline
\end{tabular}


Table 5. Comparison of OHAM-DJ solutions with exact solutions at mesh point $x=1, y=1.5, \quad \varepsilon=0.1$ (example 2).

\begin{tabular}{cccc}
\hline $\mathrm{t}$ & $u^{*}(x, y, t)=v^{*}(x, y, t)$ & $u(x, y, t)=v(x, y, t)$ & $\left|u^{*}-u\right|=\left|v^{*}-v\right|$ \\
\hline 0.01 & 0.000003917707418 & 0.000003915638782 & $2.068636 \times 10^{-9}$ \\
0.02 & 0.000004118571745 & 0.000004104638279 & $1.3933466 \times 10^{-8}$ \\
0.03 & 0.000004329734519 & 0.000004293637776 & $3.6096743 \times 10^{-8}$ \\
0.04 & 0.000004551723744 & 0.000004482637274 & $6.9086470 \times 10^{-8}$ \\
0.05 & 0.000004785094494 & 0.000004671636770 & $1.13457724 \times 10^{-7}$ \\
0.06 & 0.000005030430303 & 0.000004860636268 & $1.69794035 \times 10^{-7}$ \\
0.07 & 0.000005288344616 & 0.000005049635766 & $2.38708850 \times 10^{-7}$ \\
0.08 & 0.000005559482332 & 0.000005238635264 & $3.20847068 \times 10^{-7}$ \\
0.09 & 0.000005844521423 & 0.000005427634761 & $4.16886662 \times 10^{-7}$ \\
0.10 & 0.000006144174603 & 0.000005616634259 & $5.27540344 \times 10^{-7}$ \\
\hline
\end{tabular}

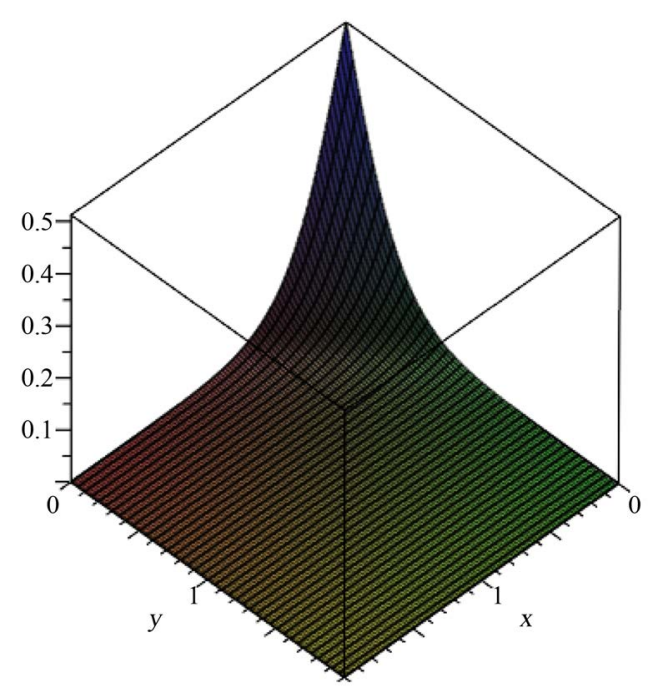

Figure 3. Approximation solutions by OHAM-DJ of example 2, $t=0.01, \quad \varepsilon=0.1$.

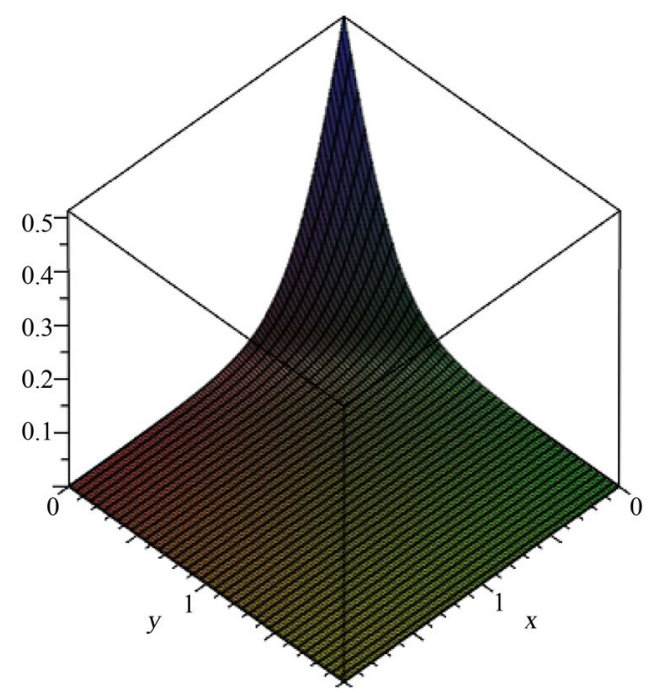

Figure 4. Exact solutions of example 2, $t=0.01, \quad \varepsilon=0.1$. 
extended to other nonlinear equations. Nutshell, OHAM-DJ is a better numerical method for solving nonlinear equations.

\section{Acknowledgements}

This paper was funded by King Abdulaziz City for Science and Technology (KACST) in Saudi Arabia. The authors therefore, thank them for their full collaboration.

\section{References}

[1] Bateman, H. (1915) Some Recent Researches on the Motion of Fluids. Monthly Weather Review, 43, 163-170. http://dx.doi.org/10.1175/1520-0493(1915)43<163:SRROTM>2.0.CO;2

[2] Burgers, J.M. (1948) A Mathematical Model Illustrating the Theory of Turbulence. Advances in Applied Mechanics, 1, 171-199. http://dx.doi.org/10.1016/S0065-2156(08)70100-5

[3] Wazwaz, A.M. (2002) Partial Differential Equations: Methods and Applications.

[4] Naghipour, A. and Manafian, J. (2015) Application of the Laplace Adomian Decomposition and Implicit Methods for Solving Burgers' Equation. TWMS Journal of Pure and Applied Mathematics, 6, 68-77.

[5] Logan, J.D. (1994) An Introduction to Nonlinear Partial Differential Equations. Wily-Interscience, Balkema.

[6] Srivastava, V.K., Ashutosh and Tamsir, M. (2013) Generating Exact Solution of Three Dimensional Coupled Unsteady Nonlinear Generalized Viscous Burgers’ Equations. International Journal of Mathematical Sciences, 5, 1-13.

[7] Fletcher, C.A. (1983) Generating Exact Solutions of the Two-Dimensional Burgers’ Equations. International Journal for Numerical Methods in Fluids, 3, 213-216. http://dx.doi.org/10.1002/fld.1650030302

[8] Fletcher, C.A.J. (1983) A Comparison of Finite Element and Finite Difference Solutions of the One- and Two-Dimensional Burgers' Equations. Journal of Computational Physics, 51, 159-188. http://dx.doi.org/10.1016/0021-9991(83)90085-2

[9] Kutluay, S., Bahadir, A.R. and Ozdes, A. (1999) Numerical Solution of One-Dimensional Burgers Equation: Explicit and Exact-Explicit Finite Difference Methods. Journal of Computational and Applied Mathematics, 103, 251-261. http://dx.doi.org/10.1016/S0377-0427(98)00261-1

[10] Mittal, R.C. and Sighal, P. (1996) Numerical Solution of Periodic Burgers Equation. Indian Journal of Pure and Applied Mathematics, 27, 689-700.

[11] Khan, M. (2014) A Novel Solution Technique for Two Dimensional Burger's Equation. Alexandria Engineering Journal, 53, 485-490. http://dx.doi.org/10.1016/j.aej.2014.01.004

[12] Ahmad, J., Bibi, Z. and Noor, K. (2014) Laplace Decomposition Method Using He’s Polynomial to Burgers Equation. Journal of Science and Arts, 14, 131.

[13] M.A. Abdou and A.A. Soliman. Variational iteration method for solving burger's and coupled burger's equations. Journal of Computational and Applied Mathematics, 181(2):245-251, 2005. http://dx.doi.org/10.1016/j.cam.2004.11.032

[14] Desai, K.R. and Pradhan, V.H. (2012) Solution of Burger's Equation and Coupled Burger's Equations by Homotopy Perturbation Method. International Journal of Engineering Research and Applications (IJERA), 2, 2033-2040.

[15] Young, D.L., Fan, C.M., Hu, S.P. and Atluri, S.N. (2008) The Eulerian-Lagrangian Method of Fundamental Solutions for Two-Dimensional Unsteady Burgers’ Equations. Engineering Analysis with Boundary Elements, 32, 395-412. http://dx.doi.org/10.1016/j.enganabound.2007.08.011

[16] Marinca, V. and Herisanu, N. (2015) The Optimal Homotopy Asymptotic Method: Engineering Applications. Springer International Publishing, Gewerbestrasse. http://dx.doi.org/10.1007/978-3-319-15374-2

[17] Marinca, V., Herisanu, N. and Nemes, I. (2008) Optimal Homotopy Asymptotic Method with Application to Thin Film Flow. Open Physics, 6, 648-653. http://dx.doi.org/10.2478/s11534-008-0061-x

[18] Marinca, V., Herisanu, N., Bota, C. and Marinca, B. (2009) An Optimal Homotopy Asymptotic Method Applied to the Steady Flow of a Fourth-Grade Fluid Past a Porous Plate. Applied Mathematics Letters, 22, 245-251. http://dx.doi.org/10.1016/j.aml.2008.03.019

[19] Marinca, V. and Herisanu, N. (2008) Application of Optimal Homotopy Asymptotic Method for Solving Nonlinear Equations Arising in Heat Transfer. International Communications in Heat and Mass Transfer, 35, 710-715. http://dx.doi.org/10.1016/j.icheatmasstransfer.2008.02.010

[20] Gupta, A.K. and Ray, S.S. (2014) Comparison between Homotopy Perturbation Method and Optimal Homotopy Asymptotic Method for the Soliton Solutions of Boussinesq-Burger Equations. Computers \& Fluids, 103, 34-41. http://dx.doi.org/10.1016/j.compfluid.2014.07.008 
[21] Kashkari, B.S. (2014) Application of Optimal Homotopy Asymptotic Method for the Approximate Solution of Kawahara Equation. Applied Mathematical Sciences, 8, 875-884.

[22] Ullah, H., Nawaz, R., Islam, S., Idrees, M. and Fiza, M. (2015) The Optimal Homotopy Asymptotic Method with Application to Modified Kawahara Equation. Journal of the Association of Arab Universities for Basic and Applied Sciences, 18, 82-88. http://dx.doi.org/10.1016/j.jaubas.2014.05.004

[23] Iqbal, S., Idrees, M., Siddiqui, A.M. and Ansari, A.R. (2010) Some Solutions of the Linear and Nonlinear Klein-Gordon Equations Using the Optimal Homotopy Asymptotic Method. Applied Mathematics and Computation, 216, 28982909. http://dx.doi.org/10.1016/j.amc.2010.04.001

[24] Nawaz, R., Ullah, H., Islam, S. and Idrees, M. (2013) Application of Optimal Homotopy Asymptotic Method to Burger Equations. Journal of Applied Mathematics, 2013, Article ID: 387478. http://dx.doi.org/10.1155/2013/387478

[25] Ishaq, M. and Haq, S. (2014) An Efficient Numerical Method for the Solution of Coupled Two Dimensional Burger's Equations. World Applied Sciences Journal, 29, 277-286.

[26] Jafari, H. and Gharbavy, S. (2012) The Solution of KDV-Burgers Equation by the Optimal Homotopy Asymptotic Method. Studies in Nonlinear Sciences, 3, 1-7.

[27] Islam, S., Nawaz, R., Arif, M. and Shah, I.A. (2012) Application of Optimal Homotopy Asymptotic Method to the Equal Width Wave and Burger Equations. Life Science Journal, 9, 2380-2386.

[28] Daftardar-Gejji, V. and Jafari, H. (2006) An Iterative Method for Solving Nonlinear Functional Equations. Journal of Mathematical Analysis and Applications, 316, 753-763. http://dx.doi.org/10.1016/j.jmaa.2005.05.009

[29] Bhalekar, S. and Daftardar-Gejji, V. (2011) Convergence of the New Iterative Method. International Journal of Differential Equations, 2011, Article ID: 989065. http://dx.doi.org/10.1155/2011/989065

[30] Ali, J. (2013) Analysis and Application of Optimal Homotopy Asymptotic Method and the Use of Daftardar Jaffery Polynomials. Doctoral Dissertation, Islamia College Peshawar, Peshawar.

[31] Ali, J., Shah, S., Islam, S. and Khan, H. (2013) Application of Optimal Homotopy Asymtotic Method with DaftardarJaffari Polynomials to Non-Linear Differential Equations. World Applied Sciences Journal, 28, 1456-1462.

[32] Shah, Z., Nawaz, R., Shah, S., Shah, S.I.A. and Shah, M. (2016) Use of the Daftardar-Jafari Poly-Nomials in Optimal Homotopy Asymptotic Method for the Solution of Linear and Nonlinear Klein-Gordon Equations. Journal of Applied Environmental and Biological Sciences, 6, 71-81.

[33] Pathak, S.P. and Singh, T. (2015) Solution of Coupled Non-Linear System by Optimal Homotopy Analysis Method. International Journal of Conceptions on Computing and Information Technology, 3, 49.

[34] Yu, X., Zhao, G. and Zhang, R. (2011) The New Numerical Method for Solving the System of Two-Dimensional Burgers’ Equations. Computers \& Mathematics with Applications, 62, 3279-3291. http://dx.doi.org/10.1016/j.camwa.2011.08.044

\section{Submit or recommend next manuscript to SCIRP and we will provide best service for you:}

Accepting pre-submission inquiries through Email, Facebook, LinkedIn, Twitter, etc.

A wide selection of journals (inclusive of 9 subjects, more than 200 journals)

Providing 24-hour high-quality service

User-friendly online submission system

Fair and swift peer-review system

Efficient typesetting and proofreading procedure

Display of the result of downloads and visits, as well as the number of cited articles

Maximum dissemination of your research work

Submit your manuscript at: http://papersubmission.scirp.org/ 